\title{
Propagation characteristic of laser- generated visco-elastic Rayleigh-like waves in stratified half-space
}

\author{
Q. B. $\operatorname{Han}^{1 *}$, J. G. Shen², X. P. Jiang ${ }^{1}$, C. Yin ${ }^{1}$, J. Jia ${ }^{1}$ and C. P. Zhu ${ }^{1}$
}

\begin{abstract}
This paper reports on a study of the propagation characteristics of visco-elastic, Rayleigh-like waves in stratified half-space structures. Beginning with the Kelvin model, the characterization equation and the normal displacement of visco-elastic Rayleigh waves in stratified half-space structures are derived and the influence of the visco-elastic modulus on dispersion and attenuation is discussed. Theoretical calculations show that the attenuation-frequency curves perfectly match the phase-frequency curves. The effect of visco-elasticity on the attenuation of the Rayleigh-like wave is larger than its effect on dispersion. For "weak viscosity," the attenuation is directly proportional to the viscosity modulus and the shear viscosity has a greater impact on the dispersion curves than does the bulk viscosity. The transient response of a visco-elastic Rayleigh wave is also simulated by means of Laplace and Hankel inversion transforms. The results are in good agreement with the theoretic predictions. It is believed the paper's results and conclusions will provide insights and guidance for estimating visco-elastic parameters and for assessing adhesive quality.
\end{abstract}

Keywords: Visco-elastic waves, Rayleigh-like waves, Laser ultrasonics

: PACS, 43.35. Cg, 43.35.ud, 43.20. Hq

\section{Introduction}

Rayleigh waves, propagating on the free surface of an elastic half space, are well known. If the medium is homogeneous and isotropic, the velocity of the Rayeleigh wave depends on the elastic constants of the medium and not on the wavelength, i.e., the Rayleigh wave is non-dispersive, and the power density of the wave decays exponentially from the surface with a characteristic penetration depth of the order of a wavelength. However, in the presence of stratified half-space, the velocity will exhibit dispersion. Stratified half-space is a kind of common material structure [1-3]. Elastic wave fields in multilayered media are of considerable interest in a variety of applications, and have, therefore, been studied extensively over the years. After Rayleigh, Love, and Stoneley, Thomson and Haskell introduced the propagator matrix method that is later focused on by many

\footnotetext{
*Correspondence: hqb0092@163.com

'College of IOT Engineering, Hohai University Changzhou, Jiangsu 213022, China

Full list of author information is available at the end of the article
}

authors. These works were carried out in the stratified elastic solid media model, and the propagator matrix technique is heuristic in many applications. The propagation of Rayleigh-like waves in a stratified half-space has been widely studied for use of nondestructive. Many years ago, Mason and Thurston [4] described surface acoustic waves (SAWs) in half substrate-coatings. Zininet et al. [5] pointed out that a pseudo-Rayleigh wave leaks energy into substrate. Lawr ultrasonics have been widely used to study the SAWs of multilayered adhesive structures [6-9], and its advantages include providing a non-contact, wide band, perfect source. Cheng et al. [10] have simulated laser-generated ultrasonic waves in a layered plate. All of the studies cited above assumed the adhesive layers are elastic solids because solidified adhesive layers closely resemble elastic solids. It should be remembered, however, that adhesive layers or coating have more attenuation than solids due to the presence of more "relaxation" or "creep."

There are two attenuation mechanisms for sound waves in layered media. The first is due to leaking 
wave where energy is leaking from one type of material to the other. Wave leaking is common when the sound wave is traveling from a solid to a liquid [11-13]. The second mechanism is material damping. Media are never perfectly elastic but always show some degree of damping that absorbs the energy of mechanical waves. Material damping is often described in the research of the waves associated with earthquakes [14, 15]. Many researchers have applied the study of waves in a stratified half-space to nondestructive evaluation (NDE) of materials. For instance, Yew et al. [16] have assessed the bonging quality of for SH waves when the adhesive layers are considered visco-elastic layers. Deschmps et al. [17] have studied acoustic emission and reflection in an anisotropic plate. Chan and Cawley [18] have discussed the effect on Lame waves brought about by viscosity through changing imaginary part of phase velocity. Bernard and Lowe [19] have studied the velocity of energy propagation in a visco-elastic plate. However, in order to simplify the calculation, most of the researches adopt Kelvin-Voigt model which directly append an imaginary part (damping) to the phase velocity and the frequency dispersion caused by visco-elasticity is ignored.

In this paper, according to the fundamental viscoelastic theory, the characterization equations for the Rayleigh waves and Rayleigh-like waves in stratified half-space structures are found by means of the Laplace/Hankel transform method. Frequency dispersion and the attenuation related to the visco-elastic modulus in a half-space, substrate-coatings, and three-layer structures are analyzed, and the transient responses of visco-elastic Rayleigh wave are simulated.

In Section 1, the theory about attenuation modes is introduced, the governing equations are derived in the transformed domain, and the equivalent elastic force sources for laser ultrasonics are discussed. In Section 2, visco-elastic Rayleigh wave propagation characteristics including dispersion, attenuation, and transient response in half-space are simulated and analyzed. In Section 3, the procedure associated with the transfer matrix approach is presented and visco-elastic Rayleigh waves in two layered structures are considered. Finally, in Section 4, three-layer adhesive structures, e.g., a half infinite metal substrate-adhesive layer-metal film, are also studied. Studying the visco-elastic (or attenuation) characteristics of waves should help us to evaluate adhesive quality and material properties. The purpose of the paper is to quantitatively analyze attenuation and dispersion and the transient response properties of Rayleigh-like waves generated by a laser and provide a theoretical basic for the determination of visco-elastic characteristics of the coatings and adhesive layers.

\section{Theory}

\subsection{Attenuation model}

Considering a three-dimension linear visco-elastic solid, the constitutive equations are [20]

$$
\sigma_{i j}=\delta_{i j} \lambda(t) * d \varepsilon_{k k}+2 \mu(t) * d \varepsilon_{i j}
$$

where $\sigma$ and $\varepsilon$ are stress and strain, respectively, $\delta_{i j}$ is Dirac delta function, $\lambda(t)$ and $\mu(t)$ are the time-dependent Lame moduli of the solid, "*:" denotes convolution, and $d$ indicates differential coefficient. The other form is expressed as

$$
\left\{\begin{aligned}
P^{\prime} S_{i j} & =Q^{\prime} e_{i j} \\
P^{\prime \prime} \sigma & =Q^{\prime \prime} e
\end{aligned}\right.
$$

where $S_{i j}=\sigma_{i j}-\frac{1}{3} \sigma_{k k} \delta_{i j}$ is stress deflection tensor, $e_{i j}$ $=\varepsilon_{i j}-\frac{1}{3} \varepsilon_{k k} \delta_{i j}$ is strain deflection tensor, $\sigma$ and $e$ are the stress symmetrical tensor and the strain symmetrical tensor, respectively; the strain is given by $\varepsilon_{i j}=\frac{1}{2}$ $\left(u_{i, j}+u_{j, i}\right), \quad u_{i, j}=\partial u_{i} / \partial x_{j}, \quad$ and $\quad P^{\prime}=\sum_{k=0}^{m^{\prime}} p_{k}^{\prime} \frac{d^{k}}{d t^{k}}, \quad P^{\prime \prime}$ $=\sum_{k=0}^{m^{\prime \prime}} p_{k}^{\prime \prime} \frac{d^{k}}{d t^{k}}, Q^{\prime}=\sum_{k=0}^{m^{\prime}} q_{k}^{\prime} \frac{d^{k}}{d t^{k}}, Q^{\prime \prime}=\sum_{k=0}^{m^{\prime \prime}} q_{k}^{\prime \prime} \frac{d^{k}}{d t^{k}}$, where $p_{k}^{\prime}$, $p_{k}^{\prime \prime}, q_{k}^{\prime}$, and $q_{k}^{\prime \prime}$ indicate the modulus in visco-elasticity mode, respectively, which are determined by material and visco-elastic mode.

\subsection{Governing equations}

The equation of motion is

$$
[\lambda(t)+2 \mu(t)] * d u_{j, j i}+\mu(t) * d u_{i, j j}=\rho \partial^{2} u_{i} / \partial t^{2}
$$

The Rayleigh wave is generated by a point pulse laser source and the coordinate axes of an isotropic plate with thickness $\mathrm{L}$ is depicted in Fig. 1a, where $z>0$ represents the stratified medium; $z=0$ is the interface between the medium and air. The surface wave excited by a point laser source has geometric attenuation symmetric about the $\mathrm{z}$-axis. When the laser irradiate on the surface of media, the Rayleigh wave is excited and propagated along radial direction. We choose point source because it is easy to focus and contribute to attenuation measurement.

In a cylindrical coordinate system, the displacement can be expressed by the potential functions $\phi$ and $\Omega_{i}$ $\left(0,-\frac{\partial \psi}{\partial r}, 0\right)$ as $u_{i}=\phi_{, i}+e_{i j k} \Omega_{k, j}$ and $\Omega_{i, i}=0$.

From Eqs. (1) and (3), the visco-elastic wave equations are given as: 


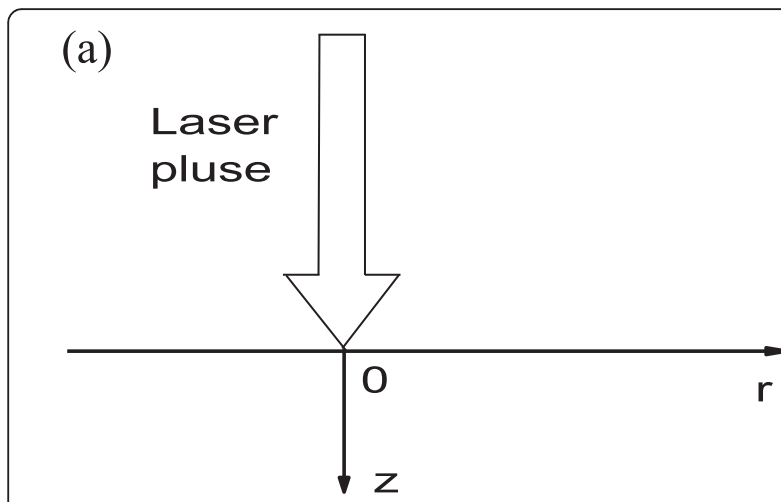

(b)

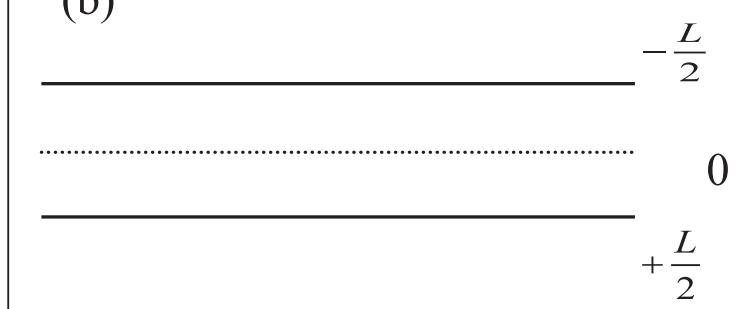

Fig 1 a Geometry used for laser generation Rayleigh wave. b The coordinate axes of an isotropic plate with thickness $H$

$$
\left\{\begin{array}{c}
\rho \frac{\partial^{2} \phi}{\partial^{2} t}-[\lambda(t)+2 \mu(t)] * d \nabla^{2} \phi=0 \\
\rho \frac{\partial^{2} \psi}{\partial^{2} t}-2 \mu(t) * d \nabla^{2} \psi=0
\end{array}\right.
$$

Applying the transform of Hankel and Laplace, the appropriate solutions of the potential functions in the transform domains can be expressed as:

$$
\left\{\begin{array}{l}
\bar{\phi}^{H_{0}}=A(p, s) e^{-\alpha z}+B(p, s) e^{\alpha z} \\
\bar{\psi}^{H_{0}}=C(p, s) e^{-\beta z}+D(p, s) e^{\beta z}
\end{array}\right.
$$

where the superscript $H_{0}$ indicates a Hankel transform of order zero and $p$ and $s$ are the space frequency and time frequency, respectively; $\alpha=\sqrt{p^{2}+\frac{s^{2}}{c_{l}^{2}}}, \quad \beta$ $=\sqrt{p^{2}+\frac{s^{2}}{c_{t}^{2}}}, c_{l}=\sqrt{\frac{\lambda(s)+2 \mu(s)}{\rho}}, c_{t}=\sqrt{\frac{\mu(s)}{\rho}}, \lambda(s)=s \lambda(s)=\frac{1}{3}$ $\left[\frac{Q^{\prime \prime}(s)}{P^{\prime \prime}(s)}-\frac{Q^{\prime}(s)}{P^{\prime}(s)}\right]$, and $\mu(s)=s \mu(s)=\frac{Q^{\prime}(s)}{2 P^{\prime}(s)}$ represent complex Lame constants associated with frequency $s ; \rho$ is density; $A, B, C$, and $D$ are constants in transform field.

The application of the Laplace transform and the Hankel transform of order zero to normal displacement $u_{z}$ as well as the stress $\tau_{z z}$ and the application of the Laplace transform and the Hankel transform of order one to the tangential displacement $u_{r}$ as well as to the stress $\tau_{r z}$, yield

$$
\begin{gathered}
\bar{u}_{r}^{H_{1}}=-p\left(\bar{\phi}^{H_{0}}+\frac{\partial \bar{\psi}^{H_{0}}}{\partial z}\right), \\
u_{z}^{H_{0}}=\frac{\partial \bar{\phi}^{H_{0}}}{\partial z}+p^{2} \bar{\psi}^{H_{0}}, \\
\bar{\tau}_{r z}^{H_{1}}=-p \mu^{*}\left(2 \frac{\partial \bar{\phi}^{H_{0}}}{\partial z}+\frac{\partial^{2} \bar{\psi}^{H_{0}}}{\partial z^{2}}+p^{2} \bar{\psi}^{H_{0}}\right), \\
\tau_{z z}^{H_{0}}=\mu^{*}\left[\left(p^{2}+\beta^{2}\right) \bar{\phi}^{H_{0}}+2 p^{2} \frac{\partial \bar{\psi}^{H_{0}}}{\partial z}\right],
\end{gathered}
$$

where the superscript $H_{1}$ indicates the Hankel transform of order one. The characterization frequency equations can be obtained by continuation of the displacement and the stress.

\subsection{The equivalent elastic force sources}

The equivalent elastic force sources generated by a point laser source on the sample surface due to the thermoelastic (ablating) effect are [21]

$$
\begin{aligned}
& \bar{\tau}_{r z}^{H_{1}}=-2 \frac{p}{s} C_{0} Q_{0} Q(s) Q(p) \\
& \bar{\tau}_{z z}^{H_{0}}=-\frac{\left(\beta^{2}+p^{2}\right)}{s \xi} C_{0} Q_{0} Q(s) Q(p)
\end{aligned}
$$

where $\xi=\sqrt{\alpha^{2}+\frac{s}{\gamma}}, \gamma$ is the thermal diffusion coefficient, and $C_{0}$ is a constant related to the thermal and elastic properties; $Q_{0}$ is the laser energy amplitude; $Q(s)$ and $Q(p)$ are the laser source function on time and space in the transform domain, respectively.

\section{Visco-elastic Rayleigh waves in half-space}

\subsection{The characterization equation}

Let us study visco-elastic Rayleigh wave in half-space firstly; the concerned conclusion will help us to understand characteristics of visco-elastic Rayleigh-like waves. Considering the fact that the two upgoing bulk wave modes vanish such that two constants $B$ and $D$ are zero in Eq. (5) in the half-space $z>0$. Substituting Eq. (5) into Eq. (6), the characterization equation of visco-elastic Rayleigh wave becomes

$$
4 p^{2} \alpha \beta-\left(\beta^{2}+p^{2}\right)^{2}=0
$$

The normal displacement of the visco-elastic Rayleigh wave in transformed domain is obtained:

$$
\bar{u}_{z}^{H_{0}}=-\alpha \frac{\Delta_{1}}{\Delta}+p^{2} \frac{\Delta_{2}}{\Delta}
$$

where

$$
\Delta=4 p^{2} \alpha \beta-\left(\beta^{2}+p^{2}\right)^{2},
$$

and 


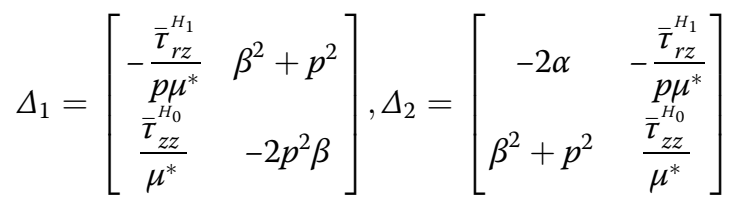

The transient response under laser source illumination can now be obtained by using the inverse Laplace and Hankel transforms as

$$
u_{z}(r, t)=\int_{0}^{\infty}\left(\int_{\alpha-i \infty}^{\alpha+i \infty} \bar{u}_{z}^{H_{0}}(p, s) e^{s t} d s\right) J_{0}(r p) p d p
$$

\subsection{Dispersion and attenuation characteristics of the visco-elastic Rayleigh wave}

From Eq. (8), the dispersion and attenuation characteristics of the visco-elasric Rayleigh wave can be obtained. The roots of the equation are complex related to frequency; with the imaginary part of the wave number is the attenuation factor in SI units of $\mathrm{Np} / \mathrm{m}$. The phase velocity of waves can be calculated from $c=\frac{\omega}{\operatorname{real}(k)}$, where $\omega$ is angular frequency and $k$ is wave number. In addition, $s=j \omega$ and $k=p$.

Because its rheological property is mainly presented by shear deformation, when the shear deformation in the Kelvin model is only considered, we have

$$
\left\{\begin{array}{c}
S_{i j}=2 \mu_{k} e_{i j}+2 \eta_{k} \cdot e_{i j} \\
\sigma=3 K e
\end{array}\right.
$$

where $\mu_{k}, \eta_{k}$, and $K$ are shear, viscous, and bulk modulus, respectively.

Combining Eq. (2) with Eq. (10) leads to

$$
\left\{\begin{array}{l}
\lambda^{\#}(s)=\mu_{k}\left(\frac{K}{\mu_{k}}-\frac{2}{3}-\frac{2}{3} K_{\eta} s\right) \\
\mu^{\#}(s)=\mu_{k}\left(1+K_{\eta} s\right)
\end{array}\right.
$$

where $K_{\eta}=\frac{\eta_{k}}{\mu_{k}}$ is the relaxation time of the visco-elastic medium (its SI unit is $s$ ), which indicates the time of strain lagging stress and $K_{\eta}=0$ represents an elastic medium, that to say, $K_{\eta}$ can describe the characteristics of viscous also. With epoxy as an example, the parameters are the bulk modulus $K=7.28 \times 10^{9} \mathrm{~kg} / \mathrm{m} . \mathrm{s}^{2}$, the shear modulus $u_{k}=2.37 \times 10^{9} \mathrm{~kg} / \mathrm{m} . \mathrm{s}^{2}$, and the thermal diffusion coefficient $\gamma=0.001 \mathrm{~cm}^{2} / \mathrm{s}$. Since the coefficient $K_{\eta}$ for a solidified epoxy is very small, two cases of $K_{\eta}=$ $10^{-10}(0.1 \eta s)$ and $10^{-9}(1 \eta s)$ were chosen in our simulation to obtain the approximate attenuation [22]. By using Eqs. (8) and (12), the dispersion curves (phase velocity versus frequency) and attenuation curves (attenuation factor versus frequency) for two cases are plotted in Fig. 2a, b which presents $K_{\eta}=0.1 \eta s$, (c) and (d) indicate $K_{\eta}=1 \eta s$.
From the dispersion curves, we find that the viscoelastic Rayleigh wave is dispersive and is not the case in (non-dispersion) elastic materials, which is due to the influence brought by viscosity. The amount of dispersion is related to the magnitude of the viscosity, and the larger the magnitude of viscosity is, the stronger the dispersion. It is also shown that the viscosity has little effect on phase velocity. When $K_{\eta}=1 \eta s$, the phase velocity of the visco-elastic Rayleigh wave increases from 1.22 to $1.26 \mathrm{~km} / \mathrm{s}$ in the frequency range $0-40 \mathrm{MHz}$ and the change in phase velocity for the case of $K_{\eta}=0.1 \eta s$ is negligible. Consequently, a sample can be regarded as non-dispersion in the case of weak viscosity, which is in correspondence with the results of Ping [23]. Accordingly, it is not very productive to study visco-elastic characteristics from the perspective of dispersion.

However, it is also shown that the attenuation of the visco-elastic Rayleigh wave increases with an increase of frequency and material viscosity. Compared with the dispersion curves, the attenuation curve variation with the frequency is more pronounced. It has been shown that the relative amplitude change in velocity versus frequency is $10 \%$ less than that of the attenuation versus frequency, [24] and the attenuation for $K_{\eta}=1 \eta s$ is higher by about one order of magnitude than for $K_{\eta}=0.1 \eta s$ at the same frequency. Thus, the elastic constants could be determined by dispersion (velocity) and the viscous constants by an attenuation curve.

Above research, we assume that the only shear viscosity, if bulk viscosity is also taken into account in the Kelvin model, the constitutive equations can be written as

$$
\begin{gathered}
\left\{\begin{array}{c}
S_{i j}=2 \mu_{k} e_{i j}+2 \eta_{k} \cdot e_{i j} \\
\sigma=3 K e+3 \eta \cdot e
\end{array}\right. \\
\left\{\begin{array}{c}
\lambda(s)=K\left(1+K_{B} s-\frac{2 \mu_{k}}{3 K}-\frac{2 \eta_{k}}{3 K} s\right) \\
\mu(s)=\mu_{k}\left(1+K_{\eta} s\right)
\end{array}\right.
\end{gathered}
$$

where $K_{B}=\frac{\eta}{K}$, and $\eta$ is the bulk viscosity modulus.

In order to investigate the influence of bulk viscosity, we suppose $K_{\eta}=0$ firstly. Let us consider the case of $K_{B}=1 \eta s$, the calculated dispersion and attenuation curves are showed in Fig. 3. Compared these results with that of $K_{\eta}=1 \eta s$ and $K_{B}=0$, it was found that the attenuation and dispersion caused by shear viscosity are much larger than that by bulk viscosity of the same frequency. In general, shear viscosity is greater than bulk viscosity for most of materials [1]. Therefore, bulk viscosity will be ignored, and only the shear viscosity will be taken in account in the following research. 


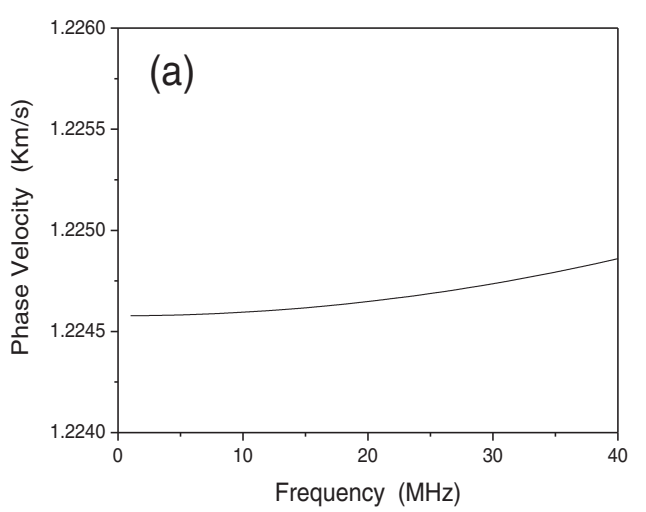

Fig 2 a Phase velocity versus frequency for epoxy half-space with $K_{n}=0.1 n$ s. b Attenuation versus frequency for epoxy half-space with $K_{\eta}=0.1 \eta$ s. $\mathbf{c}$ Phase velocity versus frequency for epoxy half-space with $K_{\eta}=1 \eta$ s. d Attenuation versus frequency for epoxy half-space with $K_{n}=1 \eta s$

\subsection{Transient response of visco-elastic Rayleigh}

To observe attenuation effect produced by viscosity intuitively, the time domain transient response of viscoelastic Rayleigh wave was simulated by means of inverse Laplace and Hankel transform. Here, we use laser ultrasonic to simulate the transient response of visco-elastic Rayleigh wave, because pulsed laser sources provide a nondestructive, non-contact means of wide bandwidth
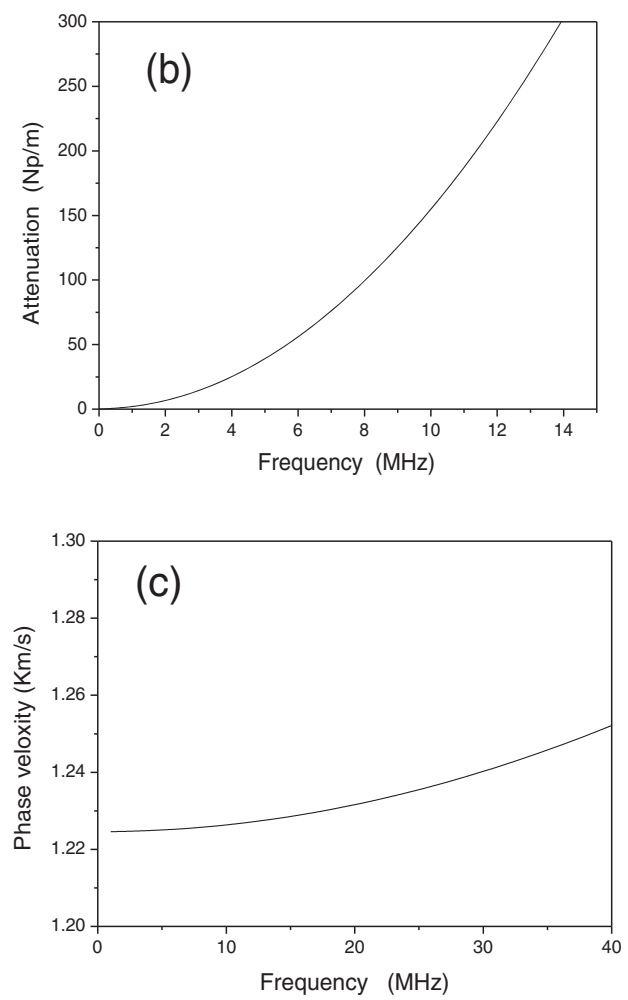
acoustic wave generation, especially, its good repetition will be good for attenuation measurement; point excited source is adopted to focus easily on producing higherfrequency wave for attenuation measurement.

The time domain transient response of visco-elastic Rayleigh waves is related to excited source. Here, the laser source function is chosen to be
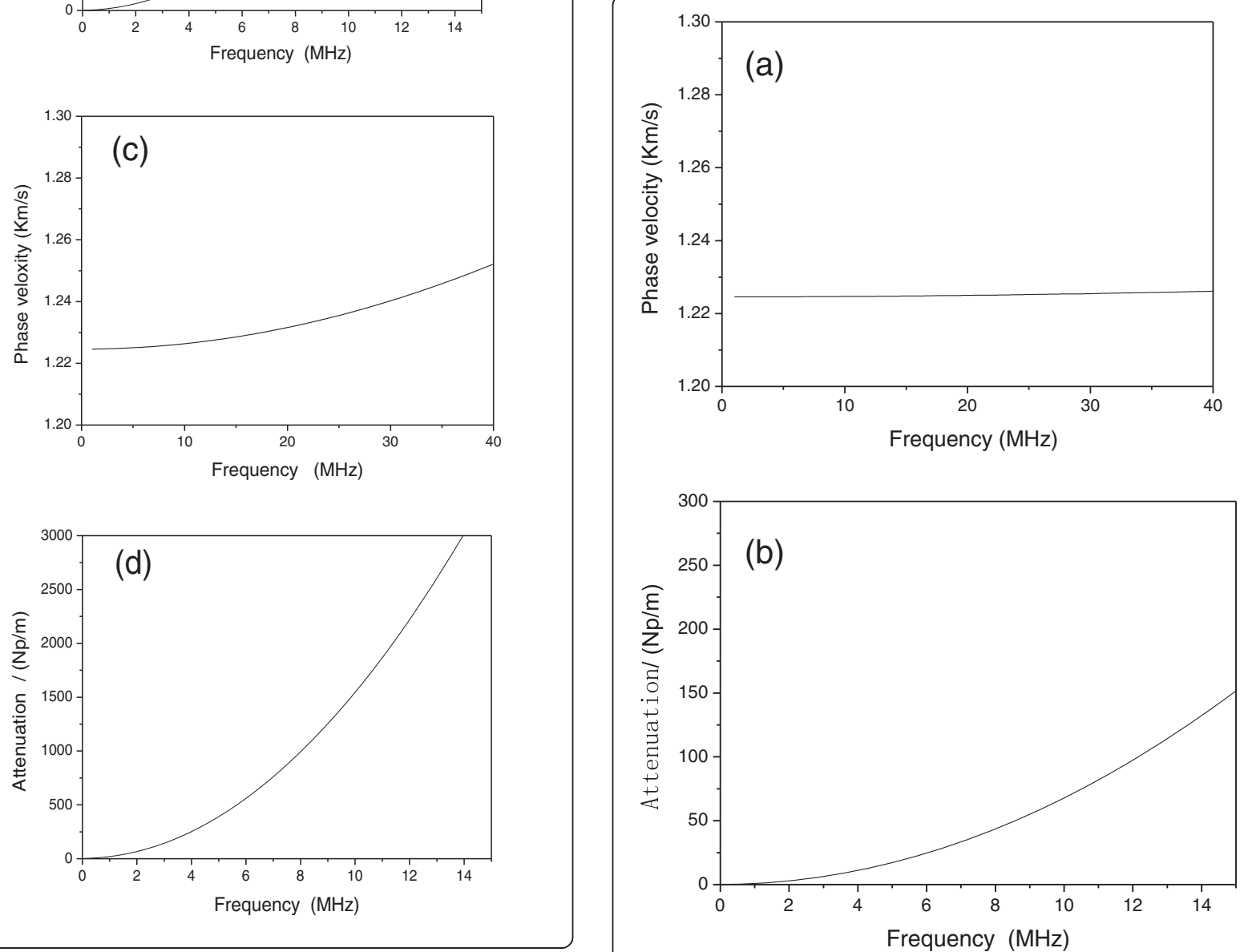

Fig 3 a Phase velocity versus frequency for epoxy half-space with $K_{B}=$ $1 \eta$ s. b Attenuation versus frequency for epoxy half-space with $K_{B}=1 \eta$ s 


$$
Q(r, t)=Q_{0}\left[\frac{2}{R^{2}} \exp \left(-2 \frac{r^{2}}{R^{2}}\right)\right]\left[\frac{t}{t_{0}^{3}} \exp \left(-\frac{t}{t_{0}}\right)\right] \delta(z)
$$

Its transform is given by

$$
\bar{Q}^{H_{0}}=Q_{0} \exp \left(-\frac{R^{2} p^{2}}{8}\right) \times \frac{1}{\left(1+t_{0} s\right)}
$$

where $t_{0}$ is the laser pulse rise time (in our calculation $\left.t_{0}=10 \eta s\right), R$ is the laser pulse Gaussian radius $(R=$ $0.1 \mathrm{~mm}$ ), and $Q_{0}$ is the absorbed laser energy. This source has been shown to accurately represent the stress field induced by a laser in a number of practical cases [21]. The representation is subject to the following assumptions: the heating is localized to the surface layer, the point of observation is outside of the volume defined by significant thermal diffusion, and the optical energy is converted to heat close to the irradiated boundary. The first and second assumptions hold if the thermal diffusion length is sufficiently less than the top layer thickness and the source to receiver distance, respectively. The third assumption holds as long as the top layer material is a strong absorber at the generation laser wavelength.

The Fourier-Bessel or Hankel transform is frequently used as a tool for solving numerous scientific problems and becomes very useful in the analysis of wave field. Equation (10) is used to calculate the transient response in stratified half-space. For a multilayered plate, there are an infinite number of singularities for particular frequency values in the integrand of the equations. These values correspond to an infinite number of poles associated with the zeroes of the Rayleigh-like frequency equation that relates frequency and wave number for guided waves in a layered plate. Since all of the poles are simple poles for a layered plate, the integral is carried out along a contour that is not on the imaginary axis so that the singularities can be avoided. Here, we apply the Secada method to inverse Laplace and Hankel transforms [25] to Eq. (9); this method uses an integral representation of Bessel functions for the transform as a weighted integral of Fourier components of the output function, by the means of computer and FFT technology, the inversing transient responses of the displacement can be obtained quickly. The transient responses of the Rayleigh wave at distance $r=5 \mathrm{~mm}$ and $r=7 \mathrm{~mm}$ for different $K_{\eta}$ are shown in Figs. 4 and 5. Again, $K_{\eta}=0$ implies the case of an elastic body.

The profiles of the Rayleigh wave are similar to that in an elastic medium; there are three waveforms that can be identified by their arrival times: the first is the
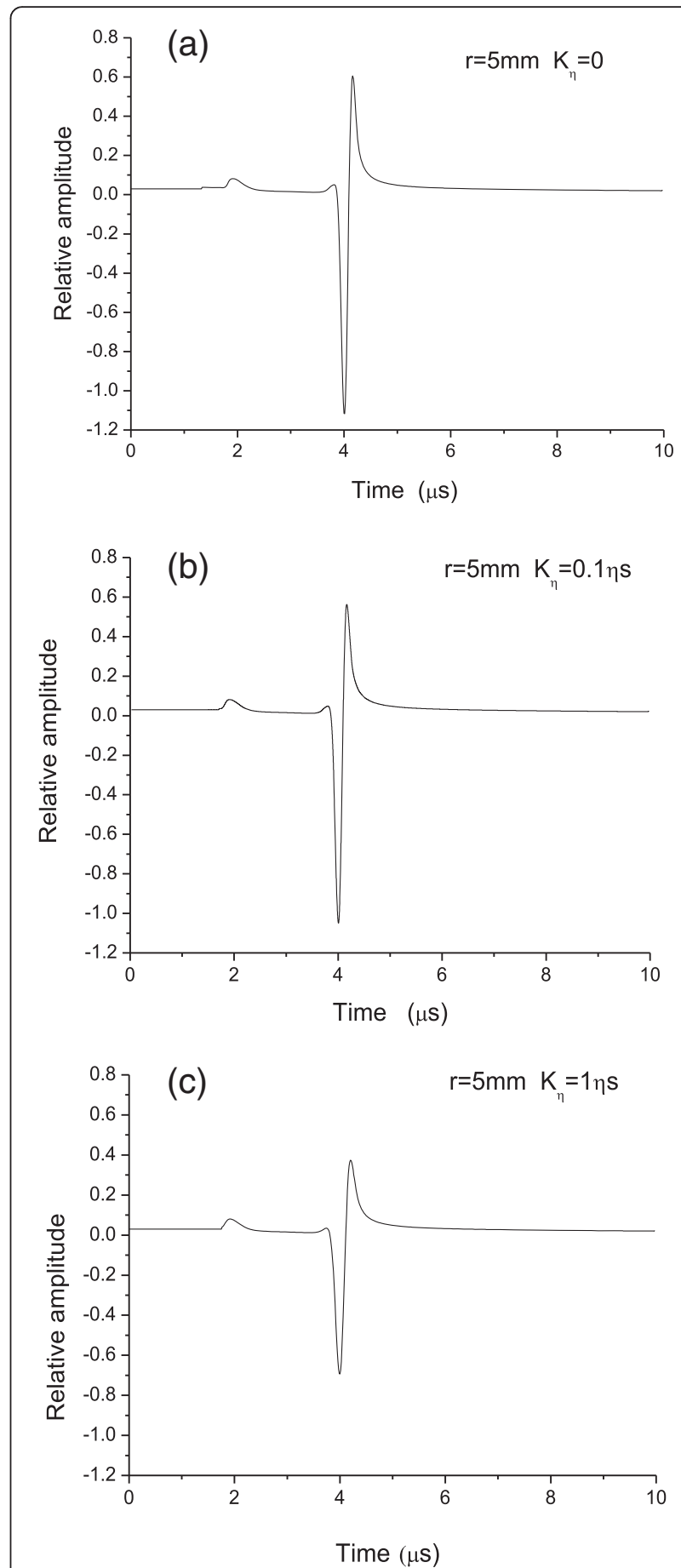

Fig 4 Transient response of the Rayleigh wave on epoxy half-space at $r=5 \mathrm{~mm}$ for $\mathbf{a} K_{n}=0, \mathbf{b} K_{\eta}=0.1 \eta \mathrm{s}$, and $\mathbf{c} K_{\eta}=1 \eta \mathrm{s}$

lateral (or Head) wave which propagates along surface with longitudinal velocity of medium, the second is shear lateral wave and very weak. The amplitude of Rayleigh wave is greatest and can be clearly identified. It is found that the transient response amplitude of the Rayleigh wave decreases with increase in the 


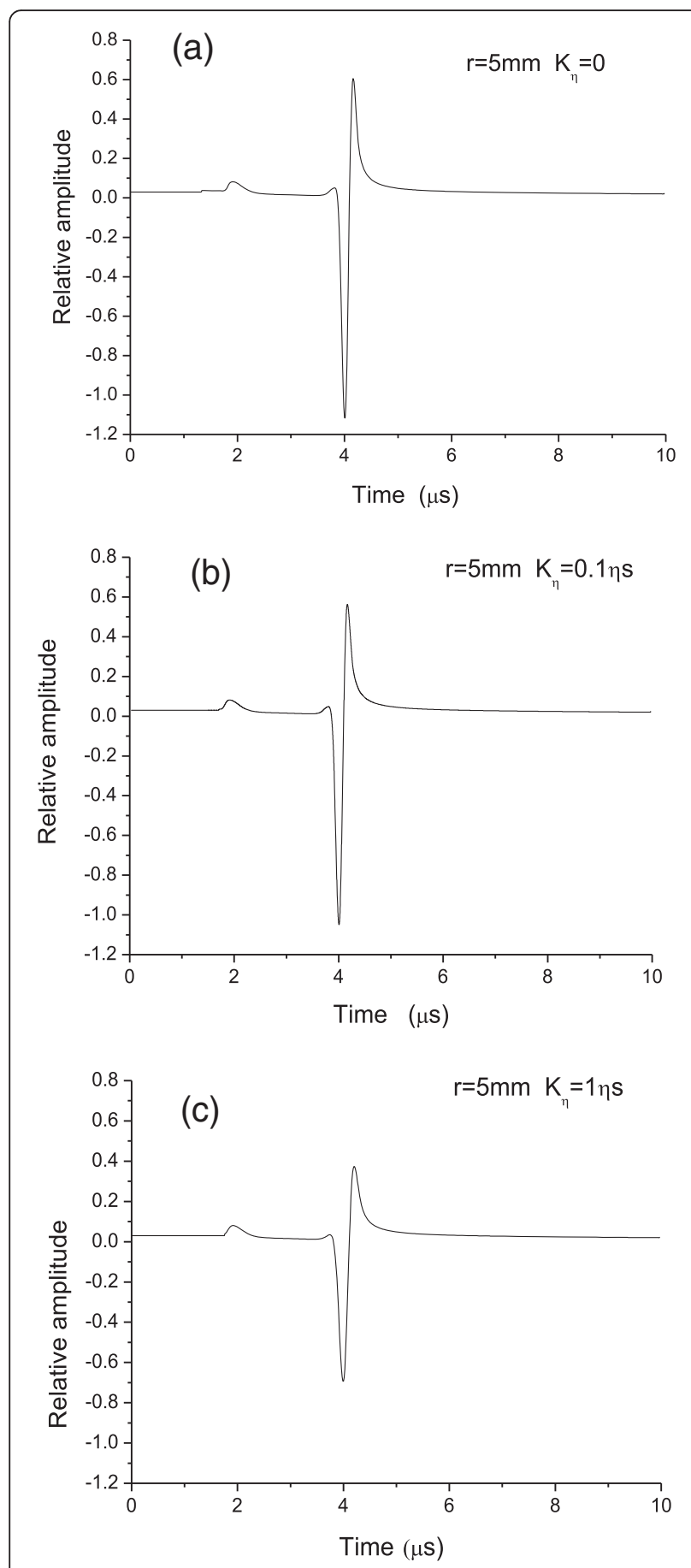

Fig 5 Transient response of Rayleigh wave on epoxy half-space at $r=7 \mathrm{~mm}$ for $\mathbf{a} K_{\eta}=0$, b $K_{\eta}=0.1 \eta \mathrm{s}$, and $\mathbf{c} K_{\eta}=1 \eta \mathrm{s}$

propagation distance for the same viscosity, which may attribute to the presence of geometric attenuation for cylindrical diffusion propagation. However, at the propagation distance, the amplitude of the Rayleigh wave decreases with increase of viscosity, from which the effect of viscosity on Rayleigh waves is clearly seen.
Table 1 The simulation parameters of two layers adhesive structures

\begin{tabular}{lllll}
\hline Materials & $c_{1}(\mathrm{~km} / \mathrm{s})$ & $c_{\mathrm{t}}(\mathrm{km} / \mathrm{s})$ & Thickness $(\mathrm{mm})$ & $\rho\left(\mathrm{g} / \mathrm{cm}^{3}\right)$ \\
\hline $\mathrm{Al}$ & 6.32 & 3.13 & $\infty$ & 2.72 \\
Epoxy & 2.73 & 1.30 & 0.1 & 1.40 \\
\hline
\end{tabular}

\section{Substrate coating structure}

\subsection{The transfer matrix}

For the layered media, the transfer matrix method introduced by Lowe [26] is adopted in this paper. Consider a single plate, which is homogenous, isotropic, and linearly elastic layer of thickness L. The origin of the coordinate system is taken to be the center of the plate, as seen in Fig. 1b, at $z=\mp \frac{L}{2}$. We define the center of every plate corresponding to $z=0 ; z=\mathrm{Ln} / 2$ indicates the surface of the $n$th plate. From Eqs. (5) and (6), we obtain the transfer relation of the stress-displacement vectors at the two sides of a plate as

$$
\left[\begin{array}{c}
\bar{u}_{r}^{H_{1}} \\
-\frac{p}{H_{0}} \\
-\frac{\bar{\tau}_{r z}^{H_{1}}}{p} \\
\bar{\tau}_{z z}^{H_{0}}
\end{array}\right]_{z=\frac{L}{2}}=M\left[\begin{array}{c}
\bar{u}_{r}^{H_{1}} \\
p \\
\bar{u}_{z}^{H_{0}} \\
-\frac{\bar{\tau}_{r z}^{H_{1}}}{p} \\
\bar{\tau}_{z z}^{H_{0}}
\end{array}\right]_{z=-\frac{L}{2}}
$$

where $M=M_{B} M_{T}^{-1}$

$$
\begin{aligned}
& M_{T} \\
& =\left[\begin{array}{cccc}
1 & e^{-\alpha L} & -\beta & \beta e^{-\beta L} \\
-\alpha & \alpha e^{-\alpha L} & p^{2} & p^{2} e^{-\beta L} \\
-2 \mu \alpha & 2 \mu \alpha e^{-\alpha L} & \mu\left(p^{2}+\beta^{2}\right) & \mu\left(p^{2}+\beta^{2}\right) e^{-\beta L} \\
\mu\left(p^{2}+\beta^{2}\right) & \mu\left(p^{2}+\beta^{2}\right) e^{-\alpha L} & -2 \mu \beta p^{2} & 2 \mu p^{2} \beta e^{-\beta L}
\end{array}\right]
\end{aligned}
$$

with

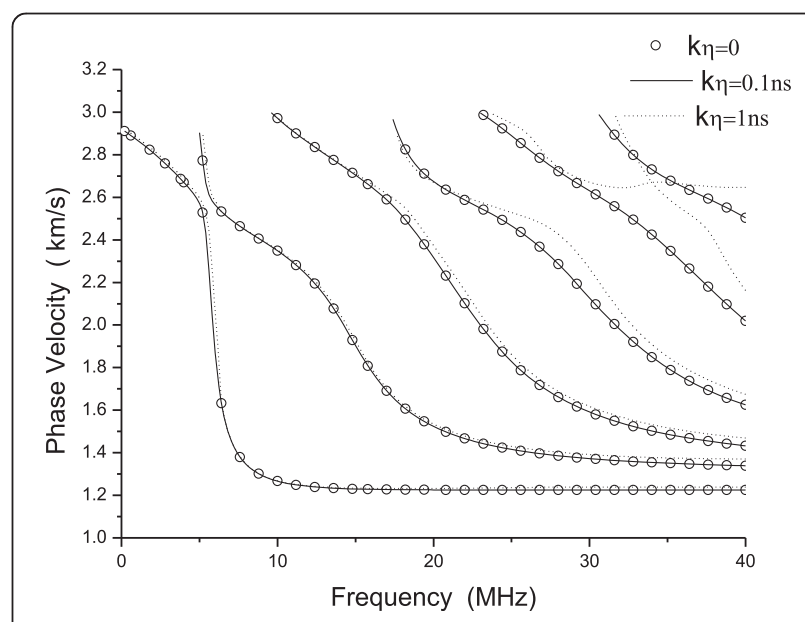

Fig 6 Comparison of dispersion curves of epoxy-Al structure for $K_{\eta}=0$ $(0), K_{\eta}=0.1 \eta \mathrm{s}(-)$, and $K_{\eta}=1 \eta \mathrm{s}(-)$ 


$$
\begin{aligned}
& M_{B} \\
& =\left[\begin{array}{cccc}
e^{\alpha L} & 1 & -\beta e^{-\beta L} & \beta \\
-\alpha e^{-\alpha L} & \alpha & p^{2} e^{-\beta L} & p^{2} \\
-2 \mu \alpha e^{-\alpha L} & 2 \mu \alpha & \mu\left(p^{2}+\beta^{2}\right) e^{-\beta L} & \mu\left(p^{2}+\beta^{2}\right) \\
\mu\left(p^{2}+\beta^{2}\right) e^{-\alpha L} & \mu\left(p^{2}+\beta^{2}\right) & -2 \mu \beta p^{2} e^{-\beta L} & 2 \mu p^{2} \beta
\end{array}\right]
\end{aligned}
$$

Now, consider a multilayered plate, consisting of $n$ layers, each of thickness $\mathrm{Hj}(j=1, \ldots, n)$. The top surface is subject to laser pulse illumination. Following the work of other authors, the laser source may be represented as an equivalent elastic boundary source consisting of distributed normal and shear loading on the plate surface as Eq. (15). Application of the continuity conditions at layer interfaces from 1 to $n$ yields

$$
\left[\begin{array}{c}
-\frac{\bar{u}_{r}^{H_{1}}}{p} \\
\bar{u}_{z}^{H_{0}} \\
-\frac{\bar{\tau}_{r z}^{H_{1}}}{p} \\
\bar{\tau}_{z z}^{H_{0}}
\end{array}\right]_{z=\frac{L_{n}}{2}}=T\left[\begin{array}{c}
-\frac{\bar{u}_{r}^{H_{1}}}{p} \\
\bar{u}_{z}^{H_{0}} \\
-\frac{\bar{\tau}_{r z}^{H_{1}}}{p} \\
\bar{\tau}_{z z}^{H_{0}}
\end{array}\right]_{z=-\frac{L_{1}}{2}}
$$

where, $T=M_{n} M_{n-1} M_{n-2} \cdots M_{j} M_{j-1} M_{j-2} \cdots M_{3} M_{2} M_{1}$, and $z=-\frac{L_{1}}{2}$ indicates the top surface. If the $n$th layer is half-space, following the same procedure leads to the layer transfer matrix,
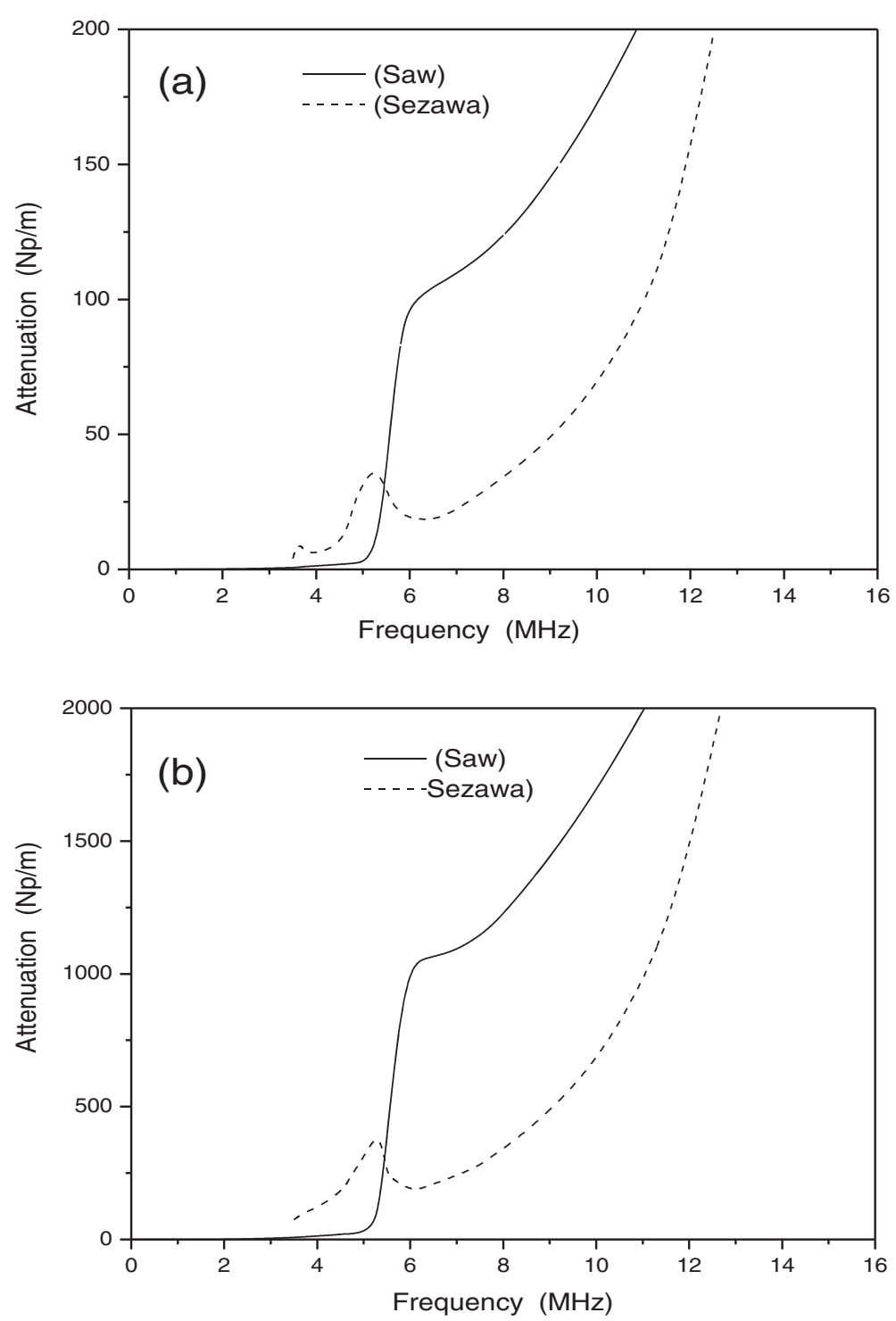

Fig 7 Attenuation versus frequency for coating-substrate structures $\mathbf{a} K_{\eta}=0.1 \eta \mathrm{s}$ and $\mathbf{b} K_{\eta}=1 \eta \mathrm{s}$ 


$$
\left[\begin{array}{c}
A_{n} \\
C_{n} \\
0 \\
0
\end{array}\right]=T\left[\begin{array}{c}
-\frac{\bar{u}_{r}^{H_{1}}}{p} \\
\bar{u}_{z}^{H_{0}} \\
-\frac{\bar{\tau}_{r z}^{H_{1}}}{p} \\
\bar{\tau}_{z z}^{H_{0}}
\end{array}\right]_{z=-\frac{L_{1}}{2}}
$$

where, $T=N_{n} M_{n-1} M_{n-2} \cdots M_{j} M_{j-1} M_{j-2} \cdots M_{3} M_{2} M_{1}$ and $N=\left[\begin{array}{cccc}1 & -\beta & 0 & 0 \\ -\alpha & p^{2} & 0 & 0 \\ -2 \mu \alpha & \mu\left(p^{2}+\beta^{2}\right) & 1 & 0 \\ \mu\left(p^{2}+\beta^{2}\right) & -2 \mu \beta p^{2} & 0 & 1\end{array}\right]$

The introduction of Eq. (7) into Eq. (19) yields the displacement at the top surface of the stratified halfspace structures in transform domain

$$
\left[\begin{array}{c}
\bar{u}_{r}^{H_{1}} \\
\bar{u}_{z}^{p}
\end{array}\right]_{z=-\frac{L_{1}}{2}}=-\left[\begin{array}{ll}
T_{31} & T_{32} \\
T_{41} & T_{42}
\end{array}\right]^{-1}\left[\begin{array}{ll}
T_{33} & T_{34} \\
T_{43} & T_{44}
\end{array}\right]\left[\begin{array}{c}
\bar{\tau}_{r z}^{H_{1}} \\
\frac{p}{H_{0}} \\
\bar{\tau}_{z z}^{H_{0}}
\end{array}\right]_{z=-\frac{L_{1}}{2}}
$$

From Eq. (20), the visco-elastic, Rayleigh-like wave equation can be derived from

$$
\operatorname{det}\left|\begin{array}{ll}
T_{31} & T_{32} \\
T_{41} & T_{42}
\end{array}\right|=0
$$

And, the normal displacement at top surface can also be obtained by means of inverting $\bar{u}_{z}^{H_{0}}$.

For visco-elastic layers, all the equations above remain true when $\lambda, \mu$ are replaced with $\lambda^{\#}, \mu^{\#}$ seen in Eqs. (11) and (12).
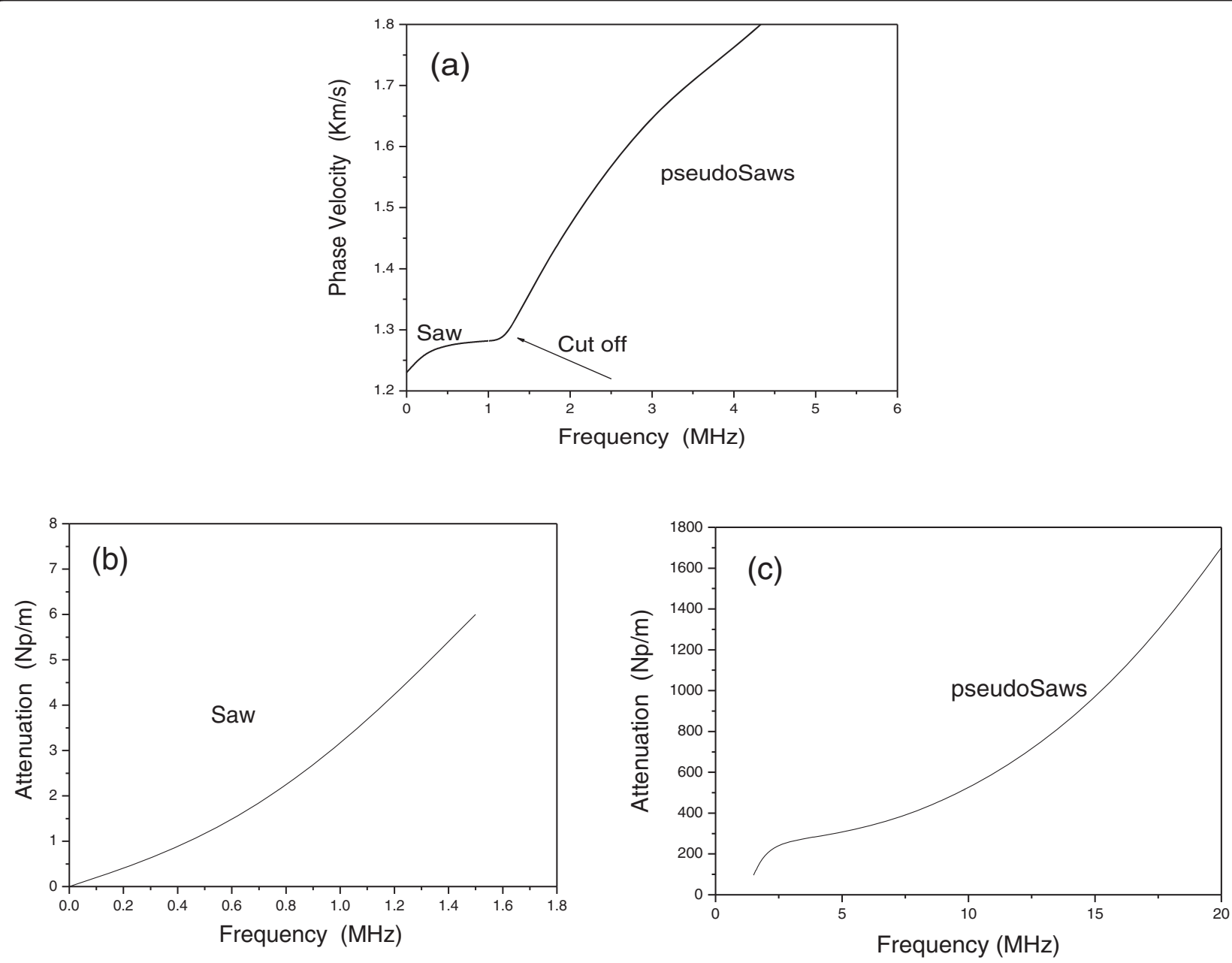

Fig 8 The substrate is epoxy, the film is Al, and the thickness of $\mathrm{Al}$ is $0.1 \mathrm{~mm}$. $K_{n}=1 \eta \mathrm{n}$. a Phase dispersion curve. $\mathbf{b}$ Attenuation curve below the cutoff frequency. c Attenuation curve above the cutoff frequency 

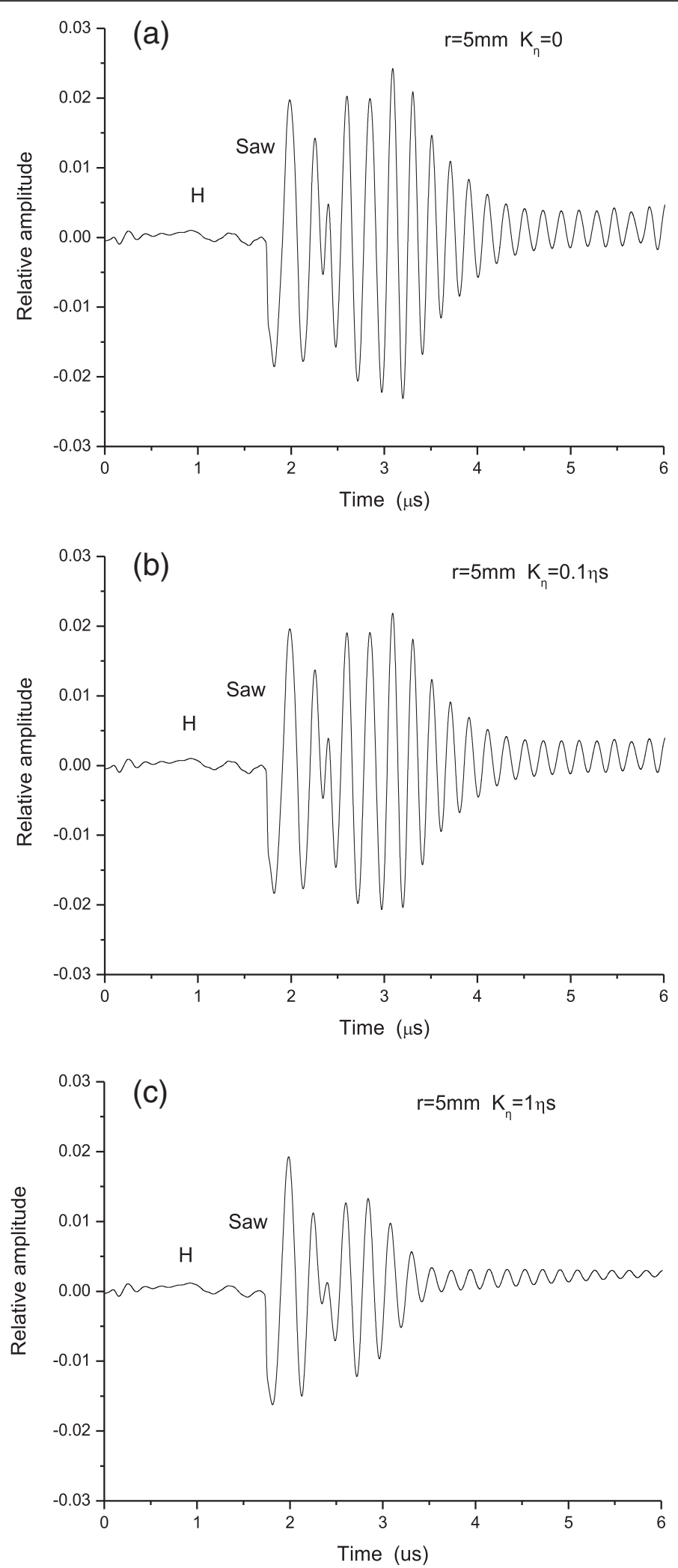

Fig 9 Transient response of the Rayleigh-like wave on epoxy_Al. Coating-substrate structure at $r=5 \mathrm{~mm}$ for a $K_{\eta}=0, \mathbf{b} K_{\eta}=0.1 \eta \mathrm{s}$, and $\mathbf{c} K_{\eta}=1 \eta \bar{s}$ 


\subsection{Slow on fast}

Where "slow on fast" means that the shear velocity of the coatings is less than that of substrate, whereas the opposite case we call "fast on slow." The elastic parameters are listed in Table 1, for the common example, aluminum and epoxy.

Considering the epoxy coatings as a visco-elastic solid, regarding the volumetric deformation as approximately elastic, and the shear deformation is visco-elastic, by using the Kelvin mode and Eq. (19), the phase velocityfrequency curves for $K_{\eta}=0, K_{\eta}=0.1 \eta s$ and $K_{\eta}=1 \eta s$ are plotted in Fig. 6. Many wave modes exist, and they are dispersive. The first two modes are named the SAW mode and the SEZAWA mode. For the SAW mode, the Rayleigh velocity starts at the Rayleigh velocity of the substrate and slopes downward approaching the Rayleigh velocity of the coating. It can also be seen that the curves are almost a superposition in the low-frequency range, implying viscosity has little influence on dispersion, and the effect is only apparent in the higherfrequency curves with the attendant increase in viscosity. It should be emphasized that f*h (frequency thickness accumulation) is not applied in constructing the curves because of an inability to normalize in terms of one parameter from the frequency equation.

Figure 7a, b shows the attenuation-frequency curves when $K_{\eta}=1 \eta s$ and $K_{\eta}=0.1 \eta s$. The attenuation of the SAW mode starts increasing rapidly from a particular frequency (for this example at about $5 \mathrm{MHz}$ ) which corresponds to the repaid slope range of the dispersion curves. This implies that the wave's energy is transferring from substrate to coating. The more energy concentrate in coatings, the more attenuation appears. We can also find that the attenuation is in approximately in direct ratio with the viscous modulus $\left(K_{\eta}\right)$. The SEZAWA mode is more complex than the SAW mode. An interesting phenomenon is a kind of local minimization of the attenuation which appears where the attenuation of the SAWs is rapidly increasing. The attenuation of SAW mode and SEZAWA mode shall rapidly increase in the range of the higher frequency.

\subsection{Fast on slow}

For the case of fast on slow, the situation is more complicated by the presence of the cutoff velocity occurring at the transverse wave velocity of the substrate. This situation has been discussed by P. Zinin [5] et al. using $V(z)$ curves. Here, we consider the phenomenon from the viewpoint of attenuation caused by viscosity of adhesive layers.

Suppose the substrate is epoxy and the film is aluminum with a thickness of $0.1 \mathrm{~mm}$ and $K_{\eta}=1 \eta s$, in this case, the substrate is epoxy with viscosity. The calculated frequency curve is shown in Fig. 8a. We find the SAW mode velocity increase with frequency monotonously; there exist a cutoff frequency in the phase dispersion at about $1.5 \mathrm{MHz}$ which means the shear velocity of epoxy. That to say, when velocity of Raleighlike wave surpasses that of shear in epoxy, the leak wave appears and pseudo-SAWs (leak wave) propagate and leak energy into the substrate. Figure $8 \mathrm{a}$, b shows the attenuation when SAW velocity is less and higher than shear velocity in epoxy, respectively. Since the cutoff frequency is lower, the attenuation caused by viscosity is very small while that caused by pseudo-SAWs is very large.

\subsection{Transient response}

Figure 9 shows the transient response calculated with $\bar{u}_{z}^{H_{0}}$ for the slow layer on a fast substrate at $7 \mathrm{~mm}$ from source, and the source parameters are same as Eq. (16). In the case of a slow layer on a fast substrate, the presence of the top layer decreases the surface wave velocity below that of the Rayleigh velocity of the substrate and normal dispersion is expected. We first see the arrival of the lateral longitudinal wave followed by the arrival of the surface wave. The Rayleigh-like waves are, as expected, dispersive; the high-frequency components of the surface wave travel slower than the lower-frequency components. With an increase in viscosity, the amplitude of all waves is reduced and the higher-frequency components are reduced faster than the lower-frequency ones.

\section{Three-layer adhesive structures}

Here, three-layer structures mean a half infinite metal substrate-adhesive layer-metal film; the simulation parameters are shown in Table 2 .

Choosing thicknesses of $0.1 \mathrm{~mm}-0.05 \mathrm{~m}-\infty$ and $K_{\eta}=1 \eta s$ and following the same calculation as above, we obtain the phase velocity dispersion shown in Fig. 10, and the attenuation-frequency curves are plotted in Fig. 11.

The transient response of three layers on a halfspace is different than for the substrate coating case. The SAW velocity starts at the Rayleigh velocity of the bottom substrate (aluminum) and slopes downward to the Rayleigh velocity of the adhesive layer

Table 2 Parameters of elastic epoxy and Al in three layers adhesive structures

\begin{tabular}{lllll}
\hline Materials & $c_{\mathrm{l}}(\mathrm{km} / \mathrm{s})$ & $c_{\mathrm{t}}(\mathrm{km} / \mathrm{s})$ & Thickness $(\mathrm{mm})$ & $\rho\left(\mathrm{g} / \mathrm{cm}^{3}\right)$ \\
\hline Al (upper) & 6.32 & 3.13 & 0.1 & 2.72 \\
Al (Nether) & 6.32 & 3.13 & $\infty$ & 2.72 \\
Epoxy & 2.73 & 1.30 & 0.05 & 1.40 \\
\hline
\end{tabular}



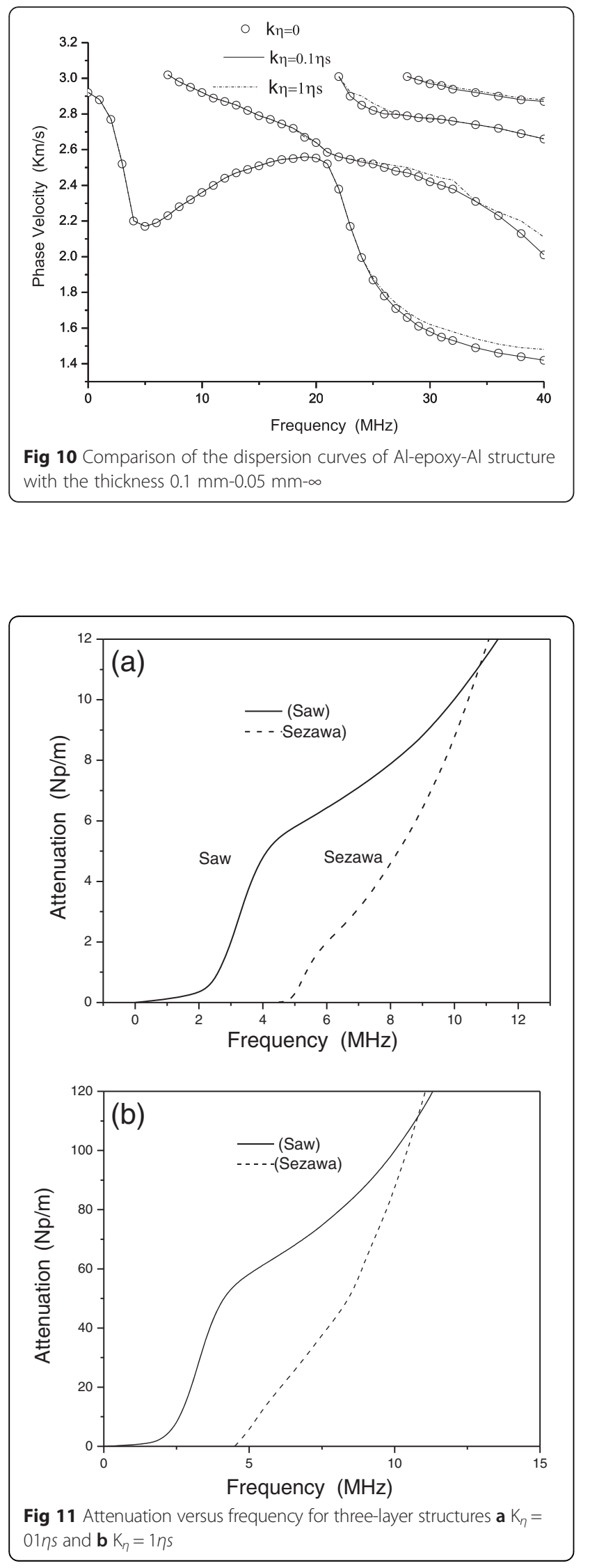

(epoxy); meanwhile, the attenuation goes through a precipitous rise, then the SAW velocity begins to increase before it reaches the velocity of the adhesive layer yet, which means the more energy go into upper medium (the elastic medium aluminum). But, the SAW velocity reduces abruptly approaching the shear velocity of the middle layer before it reaches the velocity of the upper substrate (about $20 \mathrm{MHz}$ ), which are called "energy trap" [9], which indicates that there is energy leaking into adhesive layer again, viz. the waves are "leaky waves" which should also be considered pseudo-Rayleigh waves. Figure 12 shows the transient response of a Rayleigh-like wave on aluminum-epoxy-aluminum three-layer structure. Now, the center frequency of the generated wave packet falls in the valley of the dispersion curve such that both the low- and high-frequency components of the signal travel faster than the center frequency. The higher- and lower-frequency components of the same mode are on top of each other in the time domain and what, at first inspection, appears to be the
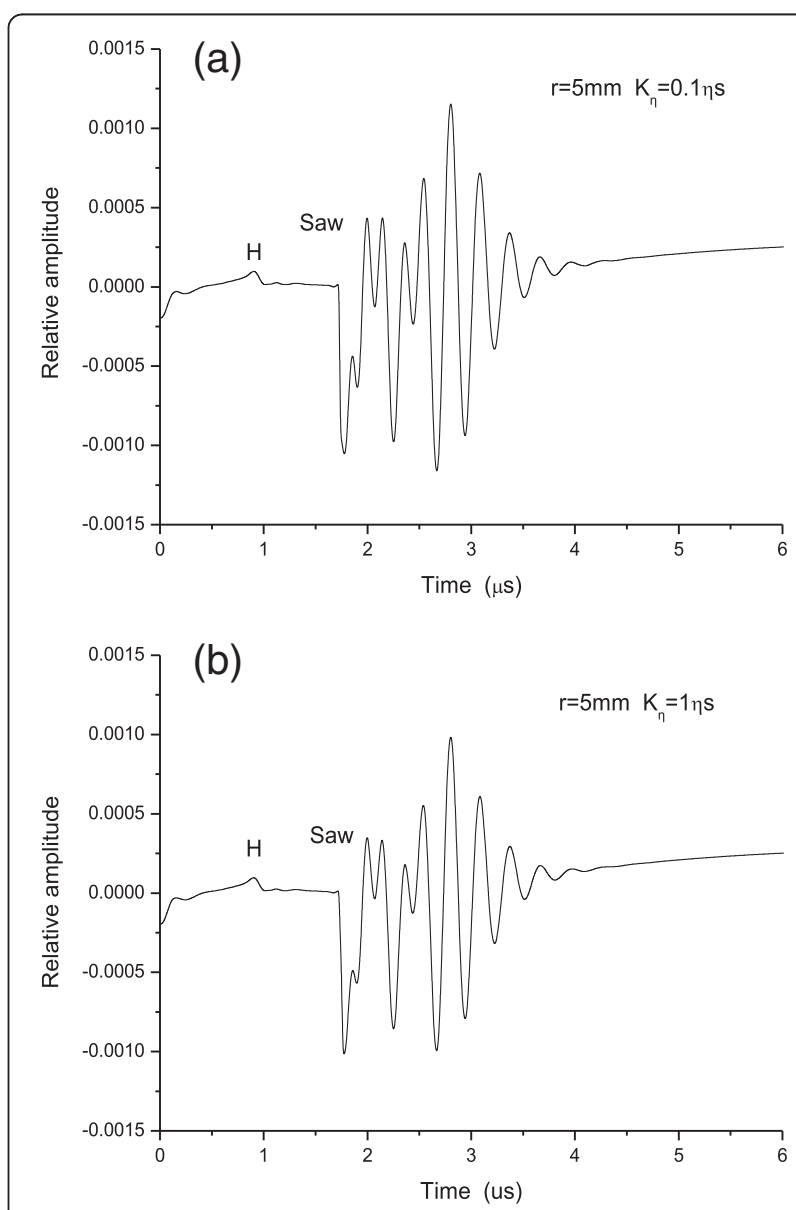

Fig 12 Transient response of the Rayleigh-like wave on Alepoxy-Al three-layer structures at $r=5 \mathrm{~mm}$ for $\mathbf{a} K_{\eta}=0.1 \eta \mathrm{s}$ and b $K_{\eta}=1 \eta s$ 
superposition of two different modes is actually the superposition of arrivals from the same mode. The first arrivals include lower- and higher-frequency waves, the middle range of frequency components arriving later which are in agreement with theoretic perdition. In addition, the amplitude of waves also decreases with viscosity.

\section{Conclusions}

In this paper, the propagation characteristics of viscoelastic, Rayleigh-like waves generated by a laser pulse are analyzed theoretically. Based on general viscoelastic theory and regarded the visco-elastic media as a Kelvin mode, the characterization frequency equations are found by means of the Laplace/Hankel transform, and transient displacement of the viscoelastic, Rayleigh-like wave is derived; the dispersion and attenuation curves due to viscosity are calculated numerically. It is shown that the dispersion of viscoelastic Rayleigh-like wave is associated with the magnitude of viscosity. In the presence of a "weak viscosity," the viscosity has little influence on phase velocity, and the attenuation of the wave is approximately proportional to the viscosity modulus. The effect of shear viscosity on attenuation is much more than that of bulk viscosity. The transient responses of the visco-elastic, Rayleigh-like wave were also simulated by the Laplace and Hankel inverse transforms, from which the effect of viscosity on the Rayleigh-like wave is clearly shown; the simulated transient response results are also in good agreement with the dispersion and attenuation curves. The approach used here was the model we develop which may provide a useful tool for the determination of the visco-elastic parameters of the material.

\section{Competing interests}

The auther declare that they have no competing interests.

\section{Acknowledgements}

This work is supported by the Natural Science foundation of China Grant Nos. 11274091 and 11574072 and the Fundamental Research Funds for the Central Universities of Hohai University No: 2011B11014.

\section{Author details}

${ }^{1}$ College of IOT Engineering, Hohai University Changzhou, Jiangsu 213022, China. ${ }^{2}$ School of Electrical Engineering and Automation, Tianjin University, Tianjin 300072, China.

Received: 14 November 2015 Accepted: 4 April 2016 Published online: 14 April 2016

\section{References}

1. K Andreas, An Evans-function approach to spectral stability of internal solitary waves in stratified fluids. J. Differ. Equ. 259, 4799-483 (2015)

2. SD Akbarov, N Illhan, A Temügan, 3D dynamics of a prestressed stratified half-space under the action of an oscillating moving load. Appl. Math. Model. 39, 1-18 (2015)
3. NO Richards, PA Erickson, An investigation of a stratified catalyst bed for small-scale hydrogen production from methanol autothermal reforming. Int. J. Hydrogen Energy 39, 18077-18083 (2014)

4. WP Mason, RN Thurston, Physical acoustics IX (Academic, New York, 1972), pp. $35-127$

5. P Zinin, O Lefeuvre, GAD Briggs, Anomalous behavior of leaky surface waves for stiffening layer near cutoff. J. Appl. Phys. 82, 1031-1035 (1997)

6. TT Wu, YH Liu, Inverse determinations of thickness and elastic properties of a bonding layer using laser-generated surface waves. Ultrasonics 37, 23-30 (1998)

7. $\mathrm{QB}$ Han, ML Qian, CP Zhu, Study of solid-solid interface waves with laser ultrasonics. Acta Phys. Sin. 56, 313-320 (2007)

8. GW Min, C Glorienx, W Lanriks, J Thoen, Investigation of titanium nitride coating by broadband laser ultrasonic spectroscopy. Chin. Phys. 11, 132-138 (2002)

9. BX Zhuang, M Yu, CQ Lan, W Xinag, Elastic waves and excitation mechanism of surface waves in multilayered media. J. Acoust. Soc. Am. 100, 3527-3538 (1996)

10. A Cheng, W Murry, JD Achenbach, Simulation of laser-generated ultrasonic waves in layered plates. J. Acoust. Soc. Am. 110, 848-855 (2001)

11. AH Nayfeh, PB Nagy, Excess attenuation of leaky Lame waves due to viscous fluid loading. J. Acoust. Soc. Am. 101, 2649-2658 (1997)

12. Z Zhu, J Wu, The propagation of Lame waves in a plate bordered with viscous liquid. J. Acoust. Soc. Am. 98, 1057-1065 (1995)

13. QB Han, ML Qian, H Wang, Investigation of liquid/solid interface waves with laser excitation and photo-elastic effect detection. J. Appl. Phys. 100, 93-101 (2006)

14. S Hestholm, S Ketcham, R Greenfield, M Moran, G McMechan, Quick and accurate Q parameterization in visco-elastic wave modeling. Geophys 71, 147-150 (2006)

15. M Jakobsen, M Chapman, Unified theory of global flow and squirt flow in cracked porous media. Geophys 74, WA65-76 (2009)

16. $\mathrm{CH}$ Yew, XW Weng, Ultrasonic SH waves to estimate the quality of adhesive bonds plate structures. J. Acoust. Soc. Am. 77, 1813-1823 (1985)

17. M Deschamps, B Hosten, Effects of visco-elasticity in the reflection and transmission ultrasonic waves by an orthotropic plate. J. Acoust. Soc. Am. 91, 2007-2015 (1992)

18. CW Chan, P Cawley, Lame waves in highly attenuative plastic plates. J. Acoust. Soc. Am. 104, 874-881 (1998)

19. A Bernard, MJS Lowe, Guided waves energy velocity in absorbing and nonabsorbing plates. J. Acoust. Soc. Am. 110, 186-196 (2001)

20. HF Cooper Jr, EL Reiss, Propagation and reflection of visco-elastic waves. J. Acoust. Soc. Am. 38, 24-34 (1965)

21. TW Murray, S Krishnaswamy, JD Achenbach, Laser generation of ultrasonic in films and coatings. Appl. Phys. Lett. 74, 716-719 (1994)

22. S Biwa, S Idekoba, N Ohno, Wave attenuation in particulate polymer composites: independent scattering/absorption analysis and comparison to measurements. Mech. Mater. 34, 671-682 (2002)

23. H Ping, Experimental verification of model for determining dispersion from attenuation. IEEE Trans. Ultrason. Ferroelectr. Freq. Control 46, 706-714 (1999)

24. D Nkemzi, The Rayleigh-Lame dispersion equations for a visco-elastic plate. Mech. Res. Commun. 20, 215-222 (1993)

25. JD Secada, Numerical evaluation of the Hankel transform. Comput. Phys. Commun. 116, 278-294 (1999)

26. MJS Lowe, Matrix techniques for modeling ultrasonic wave in multilayered media. IEEE Trans. Ultrason. Ferroelectr. Freq. Control 42, 525-542 (1995)

\section{Submit your manuscript to a SpringerOpen ${ }^{\circ}$ journal and benefit from:}

- Convenient online submission

- Rigorous peer review

- Immediate publication on acceptance

- Open access: articles freely available online

- High visibility within the field

- Retaining the copyright to your article

Submit your next manuscript at springeropen.com 